\title{
Sizanani: A Randomized Trial of Health System Navigators to Improve Linkage to HIV and TB Care in South Africa
}

\section{Citation}

Bassett, I. V., S. M. Coleman, J. Giddy, L. M. Bogart, C. E. Chaisson, D. Ross, M. M. Jacobsen, et al. 2016. "Sizanani: A Randomized Trial of Health System Navigators to Improve Linkage to HIV and TB Care in South Africa." Journal of Acquired Immune Deficiency Syndromes (1999) 73 (2): 154-160. doi:10.1097/QAl.0000000000001025. http://dx.doi.org/10.1097/QAl.0000000000001025.

\section{Published Version}

doi:10.1097/QAI.0000000000001025

\section{Permanent link}

http://nrs.harvard.edu/urn-3:HUL.InstRepos:30371169

\section{Terms of Use}

This article was downloaded from Harvard University's DASH repository, and is made available under the terms and conditions applicable to Other Posted Material, as set forth at http:// nrs.harvard.edu/urn-3:HUL.InstRepos:dash.current.terms-of-use\#LAA

\section{Share Your Story}

The Harvard community has made this article openly available.

Please share how this access benefits you. Submit a story.

Accessibility 


\title{
Sizanani: A Randomized Trial of Health System Navigators to Improve Linkage to HIV and TB Care in South Africa
}

\author{
Ingrid V. Bassett, MD, MPH, * $+\xi \|$ Sharon M. Coleman, MS, ๆ Janet Giddy, MBChB, MFamMed,\# \\ Laura M. Bogart, PhD, $\|^{* *}$ Christine E. Chaisson, MPH, $₫$ Douglas Ross, MBChB, MBA, †† \\ Margo M. Jacobsen, BA, $\neq$ Marion Robine, BA, $\neq$ Tessa Govender, BA,\# \\ Kenneth A. Freedberg, MD, MS, $*+\$ \| \neq \neq \xi \xi$ Jeffrey N. Katz, MD, $\xi \xi\|\|$ \\ Rochelle P. Walensky, MD, MPH, $+\neq \oint \| 99$ and Elena Losina, PhD $\$ \| \neq \neq \# * * *$
}

Background: A fraction of HIV-diagnosed individuals promptly initiate antiretroviral therapy (ART). We evaluated the efficacy of health system navigators for improving linkage to HIV and tuberculosis (TB) care among newly diagnosed HIV-infected outpatients in Durban, South Africa.

Methods: We conducted a randomized controlled trial (Sizanani Trial, NCT01188941) among adults ( $\geq 18$ years) at 4 sites. Participants underwent TB screening and randomization into a health system navigator intervention or usual care. Intervention participants

Received for publication December 2, 2015; accepted March 28, 2016.

From the Divisions of *Infectious Diseases; †General Medicine, Massachusetts General Hospital, Boston, MA; \$Medical Practice Evaluation Center, Massachusetts General Hospital, Boston, MA; §Harvard Medical School, Boston, MA; |Harvard University Center for AIDS Research, Harvard University, Boston, MA; ๆData Coordinating Center, Boston University School of Public Health, Boston, MA; \#McCord Hospital, Durban, South Africa; **Division of General Pediatrics, Department of Medicine, Boston Children's Hospital, Boston, MA; ††St. Mary's Hospital, Durban, South Africa; +\$Department of Epidemiology, Boston University School of

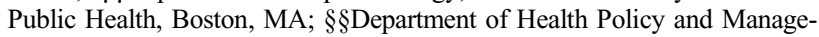
ment, Harvard School of Public Health, Boston, MA; Divisions of \|\| Rheumatology; $\uparrow \uparrow \mid$ Infectious Diseases, Brigham and Women's Hospital, Boston, MA; \#\#Department of Orthopedic Surgery, Brigham and Women's Hospital, Boston, MA; and ***Department of Biostatistics, Boston University School of Public Health, Boston, MA.

The trial was funded by the US National Institute of Mental Health R01 MH090326 (IVB). It was supported by the Harvard University Center for AIDS Research P30 AI060354 (IVB) and the National Institutes of Health R01 AI058736 (KAF) and R01 AI093269 (RPW). The contents of this publication are solely the responsibility of the authors and do not necessarily represent the official views of the US National Institute of Mental Health.

Presented in part at the 15th Conference on Retroviruses and Opportunistic Infections, February 23-26, 2015, Seattle, WA.

The authors have no conflicts of interests to disclose.

I.V.B. conceived the study and was principal investigator of the funded trial. J.G., L.M.B., D.R., K.A.F., J.N.K., R.P.W., E.L., and I.V.B. designed the study and T.G., D.R., and J.G. oversaw the trial in South Africa. M.M.J. and M.R. assisted with study protocol and manuscript preparation. S.M. C., supervised by E.L. and C.E.C., did the primary statistical analysis. All authors approved the final manuscript.

Correspondence to: Ingrid V. Bassett, MD, MPH, Massachusetts General Hospital, 50 Staniford Street, 9th Floor, Boston, MA 02114 (e-mail: ibassett@partners.org).

Copyright (C) 2016 Wolters Kluwer Health, Inc. All rights reserved. This is an open access article distributed under the terms of the Creative Commons Attribution-NonCommercial-NoDerivatives License 4.0 (CC BY-NC-ND), which permits downloading and sharing the work provided it is properly cited. The work cannot be changed in any way or used commercially. had an in-person interview at enrollment and received phone calls and text messages over 4 months. We assessed 9-month outcomes via medical records and the National Population Registry. Primary outcome was completion of at least 3 months of ART or 6 months of TB treatment for coinfected participants.

Results: Four thousand nine hundred three participants were enrolled and randomized; 1899 (39\%) were HIV-infected, with $1146(60 \%)$ ART-eligible and $523(28 \%)$ TB coinfected at baseline. In the intervention, 212 (39\% of outcome-eligible) reached primary outcome compared to $197(42 \%)$ in usual care (RR 0.93, 95\% CI: 0.80 to 1.08$)$. One hundred thirty-one (14\%) HIV-infected intervention participants died compared to $119(13 \%)$ in usual care; death rates did not differ between arms (RR 1.06, 95\% CI: 0.84 to 1.34 ). In the as-treated analysis, participants reached for $\geq 5$ navigator calls were more likely to achieve study outcome.

Conclusions: $\sim 40 \%$ of ART-eligible participants in both study arms reached the primary outcome 9 months after HIV diagnosis. Low rates of engagement in care, high death rates, and lack of navigator efficacy highlight the urgency of identifying more effective strategies for improving HIV and TB care outcomes.

Key Words: health system navigator, linkage to HIV care, HIV/TB co-infection, South Africa

(J Acquir Immune Defic Syndr 2016;73:154-160)

\section{INTRODUCTION}

Over 6.3 million people are living with HIV in South Africa, and an estimated 200,000 die of HIV each year. ${ }^{1}$ Although South Africa has the largest antiretroviral therapy (ART) program in sub-Saharan Africa, 60\% of HIV-infected South Africans are not on treatment ${ }^{2}$; those diagnosed but not yet started on ART experience high mortality rates. HIV mortality is amplified by a rampant tuberculosis (TB) epidemic. TB is the leading cause of death among HIV-infected patients in South Africa, accounting for up to $42 \%$ of deaths. ${ }^{1,3}$

We and others have documented high rates of loss to follow-up and mortality after HIV or TB diagnosis but before treatment initiation. ${ }^{4-9}$ Improving linkage to HIV and TB care before ART initiation could lead to substantial clinical and population benefits. Psychosocial factors (eg, stigma, discrimination, preference for traditional medicines), structural 
factors (eg, poverty, distance to clinic), and clinic system characteristics (eg, rigid policies, long wait times) are among the reasons given for not initiating treatment in sub-Saharan Africa ${ }^{10}$ However, few studies have evaluated interventions to improve initiation of care after diagnosis. ${ }^{11-13}$

Health system navigators have been used to help patients identify and overcome barriers to care. ${ }^{14}$ A US-based randomized trial of a health navigator delivering time-limited, in-person case management was associated with a $36 \%$ relative increase in patient clinic attendance. ${ }^{15,16}$ Our objective was to perform a randomized controlled trial using health system navigators, tailored to resource-limited settings, to evaluate their effect on ART initiation and TB treatment completion among newly diagnosed HIV-infected outpatients in Durban, South Africa.

\section{METHODS}

Described fully elsewhere, ${ }^{17}$ the Sizanani trial examined the efficacy of a health system navigator and short messaging service (SMS) reminders to attend appointments and retrieve test results on rates of linkage to and retention in HIV/TB care. Based on Anderson's model of health services utilization, we hypothesized that a health system navigator could help identify and change modifiable patient factors, including self-efficacy and social support, through strengths-based case management. ${ }^{18}$ The health system navigator engaged participants through face-to-face, telephone, and SMS communications.

\section{Study Design}

We enrolled participants between August 11, 2010 and January 16, 2013 at 4 study sites in and around Durban, South Africa: 2 hospital outpatient departments (McCord and St. Mary's Hospitals) and 2 primary health clinics. McCord Hospital had a PEPFAR-funded ART clinic, Sinikithemba, that served the urban population of greater Durban. ${ }^{19}$ Sinikithemba closed on June 15, 2012 because of loss of PEPFAR funding, and enrollment stopped at McCord's outpatient department on August 6, 2012. St. Mary's Hospital is $20 \mathrm{~km}$ west of Durban and served a poorer periurban population with its PEPFARfunded ART clinic. Participants were also enrolled at Tshelimnyama and Mariannhill, 2 nurse-driven municipal primary health clinics in the St. Mary's Hospital catchment. At study initiation, these municipal clinics offered HIV testing and referral to St. Mary's Hospital for ART initiation. As part of South Africa's decentralization of HIV care, starting October 1, 2011, these clinics began offering ART and HIV care.

The study was approved by the McCord Hospital Medical Research Ethics Committee, St. Mary's Hospital Research Ethics Committee, University of KwaZulu-Natal Biomedical Research Ethics Committee and Partners Institutional Review Board (Protocol 2011-P-001195, Boston, MA). The study was monitored by an independent Data Safety Monitoring Board.

\section{Participants}

All adults 18 years or older, English-speaking or Zuluspeaking, presenting for HIV testing and not known to be HIV-infected, were eligible for enrollment. Participants were enrolled before HIV testing to allow unbiased assessment of emotional health and social support from the earliest stage in the HIV care continuum and to allow consideration of study participation before receiving a test result that might be distressing. Children and pregnant women were excluded because they entered HIV care through a separate mechanism. Participants did not receive remuneration.

\section{Randomization}

After enrollment but before HIV testing, subjects were randomized to usual care or the health system navigator intervention. Randomization was stratified by site and gender, with blocks of varying length. Randomization assignments were accessed by the enrolling research assistant electronically through locked randomization tables in a handheld device.

\section{Procedures}

A dedicated bilingual (Zulu/English) research assistant approached patients awaiting an HIV test to assess interest and eligibility. Willing and eligible participants provided written informed consent followed by a 15-20 minute baseline questionnaire including demographics and psychosocial information regarding emotional health, social support, and selfidentified barriers to engaging in care. Participants provided their contact information and that of a friend/family member.

Participants were then randomized and presented to the HIV counselor for testing, where they received the site's usual counseling regarding their HIV test result and next steps for obtaining treatment. HIV-infected participants were offered a CD4 test and asked to return for results 2 weeks later. The HIV-infected participants were then escorted to a study TB nurse to expectorate a sputum specimen, which was sent to the microbiology laboratory at the University of KwaZulu-Natal for acid-fast bacillus smear and mycobacterial culture. Afterward, intervention arm participants were met by the health system navigator to establish a relationship, to identify perceived barriers to care, and to assess participants' coping strengths. They were then referred to a clinician for regular services. Usual care participants were referred directly to a clinician. TB-infected participants in both arms were informed of their result by the TB nurse within 48 hours of the nurse receiving positive smear or culture results.

The health system navigators were Zulu speakers with prior HIV counseling experience trained by a PhD-level social scientist and social worker in strengths-based case management. ${ }^{20}$ They were provided scripts and probes to prepare for patient interactions. The navigator met with intervention arm participants in a private space and provided focused support including discussing barriers and facilitators for entering care and described anticipated steps in the HIV/ TB care pathways. The navigator provided participants ongoing social support including 5 scheduled phone calls (weeks 1, 4, 8, 12, and 16 after enrollment) and 4 SMS reminders to retrieve test results and attend appointments, which were tailored to their progress through the HIV/TB care cascade. During each phone call, the navigator reassessed perceived barriers and coping strengths. Navigators offered participants their study mobile phone number and 
were available for questions. ${ }^{17}$ Navigators received weekly lists of participants due for phone calls; participants remained on the list for 4 weeks or until reached. Usual care participants were instructed to return to clinic for CD4 results within 2 weeks and were contacted for referral to TB care if diagnosed, but otherwise no further efforts were made to link and retain them in care by the sites and participants had no further research contacts until 9-month follow-up.

Nine months after enrollment, study staff blinded to study arm contacted participants by phone for a brief interview and collected the following from electronic and paper records at study sites: date and result of HIV test, CD4 counts, ART initiation date, and the first 3 ART dispensing dates. Mortality was obtained from clinical records, the National Population Register, and friends/family members reached during followup phone calls. We collected dates of TB treatment initiation, completion, and outcome (cured, treatment completed, treatment defaulted, treatment failure, death) from sites' TB registers. Additional outcomes were retrieved from the Department of Health's TB Control Programme.

\section{Outcomes}

The primary outcome was linkage to and initial retention in care 9 months after enrollment for living participants eligible for ART and/or TB treatment. For HIV-infected participants with negative TB testing at enrollment, the primary outcome was 3 months on ARTdocumented by initiation date and subsequent ART dispensing dates at study sites. For HIV/TB coinfected participants, linkage and retention also included 6-month TB treatment completion-documented by $\mathrm{TB}$ treatment outcome from study site TB registry or TB Control Programme database. Coinfected participants who were ART-eligible were considered to have reached the primary outcome if they reached either the HIV or TB outcome. Coinfected participants not ART-eligible were considered to have reached the primary outcome if they reached the TB outcome. We accounted for ART eligibility criteria changes in South African guidelines during the study period. ${ }^{20,21}$ ART eligibility was defined as CD4-eligible per South African guidelines at enrollment or a WHO stage 3 or 4 clinical event (including TB). Outcomes were ascertained 9 months after enrollment, allowing TBinfected individuals to complete a standard course of TB treatment and ART-eligible individuals to undergo HIV literacy training and successfully initiate ART. Mortality at 9 months was a secondary outcome. We verified death data by cross-match with the National Population Register, which encompasses $\sim 90 \%$ of deaths nationwide. ${ }^{22}$

\section{Statistical Analysis}

The study was powered to detect a $27 \%$ relative increase in reaching the primary outcome in the intervention arm with 0.052 -sided significance. Based on prior work, ${ }^{17}$ we planned to enroll 4894 participants; we anticipated 35\% HIVinfected and $21.5 \%$ eligible to reach a primary outcome.

The primary analysis was intention to treat, with participant outcomes compared according to assigned study

156 | www.jaids.com arm. Comparisons were performed using $\chi^{2}$; relative risks were calculated by log-binomial regression, with participants having at least 3 months on ART or TB treatment completion considered to have successfully reached study outcome. We assumed that participants without available data from site registries related to ART initiation or TB treatment completion did not reach study outcome. We also assessed number of call attempts by the navigator per participant and number of calls that successfully reached the participant, as a measure of fidelity to the intervention. Participants not reached at all were considered as having zero navigator phone contacts, though they received SMS per protocol. We performed a secondary "as treated" analysis in which participants were stratified by whether they received the full intervention by protocol ( $\geq 5$ calls reached) or not ( $<5$ calls reached). We used log-binomial regression to assess predictors of having $\geq 5$ successful navigator contacts. Participants who withdrew consent for telephone follow-up, but not for record review, were included.

\section{Role of the Funding Source}

The funding source had no impact on the design and implementation of the study or data interpretation. The Data Coordinating Center investigators (SMC, CEC) and lead biostatistician (EL) had full data access.

\section{RESULTS}

From August 11, 2010 to January 16, 2013, we screened 6536 people. Of those, 4954 (76\%) were eligible and 4903 (99\%) enrolled (Fig. 1). The most common reasons for ineligibility included: previous HIV diagnosis $(988,62 \%)$, $<18$ years old $(277,18 \%)$, and unwilling to share HIV/TB test results $(208,13 \%)$. A total of 1899 (39\%) enrolled subjects were newly diagnosed with HIV at enrollment of whom $967(51 \%)$ were randomized to the intervention and $932(49 \%)$ to usual care. Overall, $49 \%$ were female and mean age was 35 years (SD 10). Baseline demographic characteristics were balanced (Table 1). HIV prevalence ranged from $31 \%$ to $52 \%$ among enrollment sites.

A CD4 count was available for 1659 (87\%) HIVinfected participants $[772 / 932(83 \%)$ in usual care and $887 /$ $967(92 \%)$ in intervention]. Median CD4 was 192/ $\mu \mathrm{L}$ (IQR $72-346 / \mu \mathrm{L})$ and similar across arms: usual care participants had median CD4 200/ $\mu \mathrm{L}$ (IQR $72-363 / \mu \mathrm{L}$ ) and navigator arm participants had median CD4 186/ $\mu \mathrm{L}$ (IQR $72-332 / \mu \mathrm{L}$ ). One thousand one hundred forty-six $(60 \%)$ participants were ART-eligible based on South African guidelines at enrollment, with a median CD4 112/ $\mu$ L (IQR 47-203/ $\mu \mathrm{L}$ ).

Of the 1899 HIV-infected participants, 1685 (89\%) had available TB culture data. Three hundred sixty-nine $(22 \%)$ were TB positive by acid-fast bacillus smear and/or culture. An additional 154 participants were diagnosed with TB outside the study by testing performed on the day of enrollment (included as eligible for primary outcome): 83 chest X-ray, 36 acid-fast bacillus smear, 1 biopsy, 15 ultrasound, 1 clinical indication, 9 culture, 1 GeneXpert, and 8 unknown. Two hundred ninetythree $(30 \%)$ of intervention arm participants and $230(25 \%)$ of

Copyright (C) 2016 Wolters Kluwer Health, Inc. All rights reserved. 


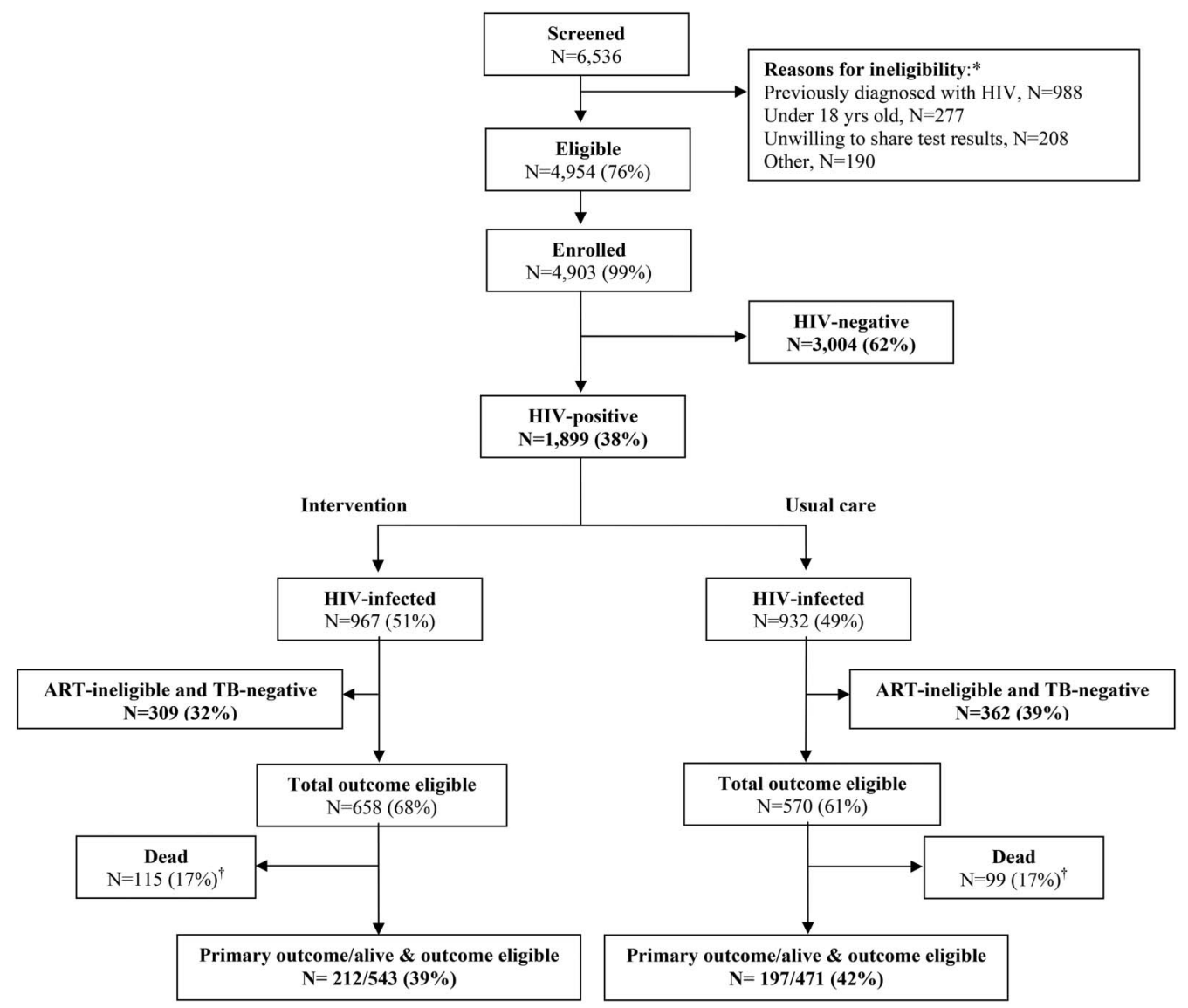

FIGURE 1. Participant flow. *Not mutually exclusive. †A total of 250 participants died. Of the 119 who died in the usual care arm, 99 were outcome eligible and 20 were not outcome eligible. Of the 131 participants who died in the intervention arm, 115 were outcome eligible and 16 were not outcome eligible.

usual care participants were coinfected with TB. Eighty-two of the HIV/TB coinfected participants were not ART-eligible, representing $4 \%$ of the HIV-infected. Forty of these were in the intervention arm and 42 in usual care.

A total of $1150(61 \%) \mathrm{HIV}$-infected participants had a valid South African ID for cross-match with the South African National Population Register. Using combined sources (National Population Register and 9-month phone calls), 250 deaths $(13 \%)$ were identified during the study.

There were 48 study withdrawals: 10 in usual care and 38 in the navigator arm. In usual care, 10 withdrew based on participant request. In the navigator arm, 2 left the country, 2 decided to use traditional remedies, 2 had not disclosed to family members, 7 did not want to discuss HIV or denied their status, 3 were already on ART and felt they did not need additional assistance, and 22 requested withdrawal. These participants were included in our analyses because they did not withdraw consent for record review.

Six hundred fifty-eight $(68 \%)$ participants in the intervention arm were ART-eligible and/or TB-infected compared with $570(61 \%)$ in usual care. In the intervention arm, 134 of the 618 (22\%) ART-eligible participants completed 3 months of ART; 119 of the 293 (41\%) TBinfected participants completed TB treatment. In usual care,
146 of the 528 (28\%) ART-eligible participants completed 3 months of ART; 101 of the 230 (44\%) TB-infected participants completed TB treatment. In both arms, some coinfected participants met both outcomes. One hundred thirty-one (14\%) HIV-infected intervention arm participants died; 119 (13\%) usual care participants died (Fig. 1). Among ARTeligible and/or TB-infected participants in the intervention arm, 212 had 3 months on ART or TB treatment completion and were alive at study completion. This represents $25 \%$ of those $\mathrm{HIV}$-infected and alive at study conclusion and 39\% of those alive and outcome-eligible in the intervention arm. Among ART-eligible and/or TB-infected participants in usual care, 197 had evidence of 3 months on ART or TB treatment completion and were alive at study completion. This is $24 \%$ of those HIVinfected and alive at study conclusion and $42 \%$ of those alive and outcome-eligible in usual care (Fig. 1 and Table 2).

The proportion of living, outcome-eligible participants reaching the composite study outcome did not differ significantly between arms (39\% in intervention and $42 \%$ in usual care; RR $0.93,95 \%$ CI: 0.80 to 1.08 ). The proportion of participants reaching a study outcome varied by site: $34 \%$ of usual care and $37 \%$ of intervention participants at McCord Hospital, $17 \%$ of usual care and $16 \%$ of intervention participants at St. Mary's Hospital, and $21 \%$ in both the usual care 
TABLE 1. Baseline Characteristics of HIV-Infected Study Participants

\begin{tabular}{|c|c|c|c|}
\hline & $\begin{array}{c}\text { Intervention } \\
(\mathrm{n}=967)\end{array}$ & $\begin{array}{c}\text { Usual Care } \\
(\mathrm{n}=932)\end{array}$ & $\begin{array}{c}\text { Overall } \\
(\mathrm{n}=\mathbf{1 8 9 9})\end{array}$ \\
\hline Age mean (SD), yrs & $35(10)$ & $35(10)$ & $35(10)$ \\
\hline \multicolumn{4}{|l|}{ Sex, $n(\%)$} \\
\hline Female & $474(49)$ & $461(49)$ & $935(49)$ \\
\hline Male & $493(51)$ & $471(51)$ & $964(51)$ \\
\hline \multicolumn{4}{|l|}{ Education, $\mathrm{n}(\%)$} \\
\hline$\leq$ Primary & 131 (14) & $139(15)$ & $270(14)$ \\
\hline $\begin{array}{l}\text { Some high school or } \\
\text { greater }\end{array}$ & $828(86)$ & $788(85)$ & $1616(86)$ \\
\hline \multicolumn{4}{|l|}{ Marital status, n(\%) } \\
\hline Never married & $778(81)$ & $759(82)$ & $1537(81)$ \\
\hline Currently married & $141(15)$ & $124(13)$ & $265(14)$ \\
\hline Divorced/widowed & $41(4)$ & $44(5)$ & $85(5)$ \\
\hline \multicolumn{4}{|l|}{$\begin{array}{l}\text { Enrolled and HIV-infected, } \\
\text { by study site, } n(\%)\end{array}$} \\
\hline McCord & 374 (39) & $355(38)$ & $729(38)$ \\
\hline St. Mary's & $389(40)$ & $375(40)$ & $764(40)$ \\
\hline Clinics & $204(21)$ & $202(22)$ & $406(21)$ \\
\hline $\begin{array}{l}\text { TB diagnosis at baseline, } \\
\mathrm{n}(\%)\end{array}$ & $293(30)$ & $230(25)$ & $523(28)$ \\
\hline $\begin{array}{l}\text { Mental Health Score } \\
\text { mean (SD) }\end{array}$ & $66(16)$ & $66(15)$ & $66(17)$ \\
\hline $\begin{array}{l}\text { Social Support Score } \\
\text { mean (SD) }\end{array}$ & $66(23)$ & $66(21)$ & $66(22)$ \\
\hline \multicolumn{4}{|l|}{$\begin{array}{l}\text { Perceived barriers to care, } \\
\mathrm{n}(\%)\end{array}$} \\
\hline Service delivery barrier & $283(29)$ & $283(31)$ & $566(30)$ \\
\hline Financial barrier & $214(22)$ & 209 (23) & $423(22)$ \\
\hline $\begin{array}{l}\text { Personal health } \\
\text { perception }\end{array}$ & $301(31)$ & $295(32)$ & $596(32)$ \\
\hline Logistical barrier & $176(18)$ & $158(17)$ & $334(18)$ \\
\hline Structural barrier & $269(28)$ & $271(29)$ & $540(29)$ \\
\hline \multicolumn{4}{|l|}{ CD4 count } \\
\hline Mean (SD) & $234(207)$ & 249 (227) & $241(217)$ \\
\hline Median (IQR) & $186(72,332)$ & $200(72,363)$ & $192(72,346)$ \\
\hline
\end{tabular}

$\mathrm{SD}$, standard deviation.

and intervention arms at the municipal clinics. With respect to closure of the McCord HIV clinic, we did not find evidence of an interaction between study arm and time of recruitment and did not find a differential effect of the intervention before and after the McCord clinic closure $(P=0.83)$.

For intervention intensity and fidelity, intervention arm participants received an average of 3.5 (SD 1.7) calls and spent an average of 17 minutes (SD 12) on the phone with the navigator. Of 967 intervention arm participants, 694 (72\%) had $\geq 5$ call attempts after enrollment as planned. Only 400 $(41 \%)$ of intervention arm participants were reached for $\geq 5$ calls, with an average of 3 (SD 4) call attempts at each unreached time point per person.

In the "as treated" analysis, $507(56 \%)$ participants in the intervention arm were reached for $<5$ calls after enrollment and 400 (44\%) for $\geq 5$ calls. These groups differed in composite outcome and mortality. Among participants

158 | www.jaids.com
TABLE 2. Study Outcomes by Randomization Group (Intention to Treat Analysis)

\begin{tabular}{|c|c|c|c|}
\hline & $\begin{array}{c}\text { Intervention } \\
\mathbf{N}(\%)\end{array}$ & $\begin{array}{c}\text { Usual Care } \\
\text { N (\%) }\end{array}$ & $\begin{array}{c}\text { Intervention vs. } \\
\text { Usual Care RR } \\
\quad(95 \% \text { CI) } \\
\end{array}$ \\
\hline \multicolumn{4}{|l|}{ Primary outcome } \\
\hline $\begin{array}{l}\text { Composite outcome*/ } \\
\text { alive and outcome } \\
\text { eligible }\end{array}$ & 212 (39) & $197(42)$ & $0.93(0.80$ to 1.08$)$ \\
\hline \multicolumn{4}{|l|}{ Secondary outcome } \\
\hline $\begin{array}{l}\text { Death (\% of HIV- } \\
\text { infected) }\end{array}$ & $131(14)$ & $119(13)$ & $1.06(0.84$ to 1.34$)$ \\
\hline
\end{tabular}

alive at study conclusion, $22 \%$ with $<5$ calls and $30 \%$ with $\geq 5$ calls reached the composite outcome compared to $24 \%$ in usual care (RR $0.95,95 \%$ CI: 0.76 to 1.19 ) and (RR 1.27 , $95 \%$ CI: 1.05 to 1.55 ), respectively. In the intervention arm, 106 participants $(21 \%)$ with $<5$ calls died during the study, and 10 participants $(2.5 \%)$ with $\geq 5$ calls died compared to 134 participants (14\%) who died in usual care (RR 1.55, 95\% CI: 1.22 to 1.95 ) and (RR $0.18,95 \%$ CI: 0.10 to 0.35 ), respectively. The proportion of subjects with successful outcomes was virtually identical at $0-2(22 \%)$ and 3-4 $(23 \%)$ calls; however, when call frequency reached $\geq 5$, the proportion reaching study outcome increased to $30 \%$.

In a secondary analysis evaluating predictors of greater contact success ( $\geq 5$ calls reached), those currently married (RR 2.09, 95\% CI: 1.16 to 3.77 ) or never married (RR 1.83 , 95\% CI: 1.02 to 3.30), enrolled at McCord Hospital (RR 1.34, 95\% CI: 1.06 to 1.71 ) or St. Mary's Hospital (RR 1.44, 95\% CI: 1.11 to 1.87 ), and reporting 0 barriers to care (RR 1.61 , $95 \%$ CI: 1.26 to 2.05 ) or $1-3$ barriers (RR $1.31,95 \%$ CI: 1.00 to 1.70 ), were more likely to have been reached for $\geq 5$ calls.

\section{DISCUSSION}

In this multicenter, randomized controlled trial, we did not find an effect of time-limited health system navigation on rates of ART initiation and TB treatment completion among people newly diagnosed with HIV in Durban, South Africa. Thirty-nine percent of alive and ART-eligible and/or TB coinfected participants in the intervention arm and $42 \%$ of participants in the usual care arm completed 3 months of ART or 6 months of TB treatment. In a secondary as-treated analysis, participants reached for the full intervention $(\geq 5$ navigator calls) were more likely to reach study outcome than participants reached for $<5$ calls or those in usual care.

Time-limited case management to promote engagement in HIV care has been effective in the United States. ${ }^{15,16,23}$ Our study is the first to evaluate efficacy of a health system navigator intervention in a resource-limited setting for improving combined HIV and TB care engagement. Our 16-week strengths-based case management intervention was conducted primarily by phone after enrollment and complemented by periodic SMS reminders. Successful US-based

Copyright (C) 2016 Wolters Kluwer Health, Inc. All rights reserved. 
studies primarily used in-person case management ${ }^{15,16,24}$ as opposed to phone and SMS. There is at least one successful US-based study that incorporated regular phone calls into its 12 -month navigation intervention. However, this included in-person case management at each HIV clinic visit. ${ }^{25}$ Sustained in-person contact may improve efficacy of a timelimited intervention.

Literature regarding the impact of SMS on HIV care is mixed. Most studies examine effects on ART adherence, ${ }^{26-30}$ whereas few examine the impact of SMS on linkage to HIV or TB care as we did; those that have reported no efficacy. ${ }^{31}$ Some studies indicate SMS may be more successful with 2way messaging and personalized content. Although we tailored SMS content to participants' stage in the HIV/TB care cascade, participants were not asked to respond to messages. ${ }^{26,27}$ Successful SMS trials have also sought to improve adherence among people already on ART, who may differ from those newly diagnosed with HIV. Newly diagnosed patients must accept their diagnosis and overcome substantial barriers to entering care. ${ }^{32}$ Patients on ART have already overcome enough of these barriers to take the steps needed for treatment. The high prevalence of HIV/TB coinfection in our population may further complicate linkage.

A US-based randomized trial that combined peer counseling with pager messaging for ART adherence showed a dose-response similar to that in our navigator arm: participants who used the pager more had better outcomes. ${ }^{30}$ In our study, participants reached for $\geq 5$ navigator calls had higher rates of achieving study outcome and lower mortality. This may indicate that higher intensity interaction with a navigator improved ART initiation and TB treatment completion and decreased mortality, or that participants more willing to answer their phones and speak with a navigator were already more likely to link to care and to survive to receive calls. Participants with fewer self-reported barriers to care were more likely to be reached for $\geq 5$ navigator calls. This suggests that those with more barriers to care, and at highest risk for poor outcomes, may need a different or more intensive intervention.

This study has several strengths and limitations. The study design was robust: participants were individually randomized before HIV testing to avoid differential acceptance rates by HIV test results. Inclusion of 4 study sites that varied geographically and by care level provided a range of participants. Finally, outcomes were based on medical record review and national registers as opposed to self-report. One explanation for the negative results is that the intervention intensity delivered was lower than intended; although $72 \%$ of intervention arm participants had $\geq 5$ call attempts, only $41 \%$ were reached for $\geq 5$ calls. It may be that the intervention intensity was insufficient to improve outcomes, or that tangible means to overcome identified care barriers (such as travel vouchers) may be needed. Some testers may have already known their HIV status and enrolled seeking care, despite our exclusion criteria; this could dilute any navigator effect. More than twice the number of participants requested study withdrawal in the intervention compared to usual care, though they represent only $2 \%$ of HIV-infected participants. This may reflect lack of willingness to engage with the navigator. We also could not distinguish lack of linkage to and retention in care from lack of documentation. Outcome ascertainment was challenging at sites that used paper-based records. Outcomes were ascertained at study sites only, so reported linkage rate might be underestimated if participants sought care elsewhere. However, we anticipate that underestimates would be balanced across arms. Ascertainment was further complicated by closure of McCord Hospital's HIV clinic, the largest enrollment site with the most robust electronic medical record. McCord patients were transferred to various regional clinics over a short period; many sought care at nonstudy sites where we were not collecting outcome data. ${ }^{6}$

Rates of engagement in HIV care remain low in Durban despite increases in HIV testing and ART availability. ${ }^{33}$ The period after diagnosis is critical for linkage to care. A health system navigator intervention complemented by SMS reminders did not show efficacy at improving ART initiation or TB treatment completion. Interventions for linkage to HIV and TB care may require higher intensity, more reliable 2-way communication between patients and providers, and provision of tangible means of overcoming barriers. Further studies are urgently needed to identify strategies for improving entry to HIV/TB care in highburden settings.

\section{ACKNOWLEDGMENTS}

The authors would like to recognize the hard work and valuable contributions of the research staff. We thank the clinical sites for their dedication to research, and we gratefully acknowledge the study participants.

\section{REFERENCES}

1. UNAIDS. South Africa HIV and AIDS Estimates. 2013. Available at: http://www.unaids.org/en/regionscountries/countries/southafrica. Accessed July 2, 2015.

2. UNAIDS. The Gap Report. 2014. Available at: http://www.unaids.org/sites/ default/files/en/media/unaids/contentassets/documents/unaidspublication/ 2014/UNAIDS_Gap_report_en.pdf. Accessed July 2, 2015.

3. World Health Organization. South Africa Tuberculosis Profile. 2013. Available at: https://extranet.who.int/sree/Reports?op=Replet\&name= \%2FWHO_HQ_Reports\%2FG2\%2FPROD\%2FEXT\%2FTBCountry Profile\&ISO2=ZA\&LAN=EN\&outtype=html. Accessed July 2, 2015.

4. Mugglin C, Estill J, Wandeler G, et al. Loss to programme between HIV diagnosis and initiation of antiretroviral therapy in sub-Saharan Africa: systematic review and meta-analysis. Trop Med Int Health. 2012;17: $1509-1520$.

5. Kranzer K, Govindasamy D, Ford N, et al. Quantifying and addressing losses along the continuum of care for people living with HIV infection in sub-Saharan Africa: a systematic review. J Int AIDS Soc. 2012;15:17383.

6. Cloete C, Regan S, Giddy J, et al. The linkage outcomes of a large-scale, rapid transfer of HIV-infected patients from hospital-based to communitybased clinics in South Africa. Open Forum Infect Dis. 2014;1:ofu058.

7. Bassett IV, Chetty S, Wang B, et al. Loss to follow-up and mortality among HIV-infected people co-infected with TB at ART initiation in Durban, South Africa. J Acquir Immune Defic Syndr. 2012;59:25-30.

8. Bassett IV, Regan S, Luthuli P, et al. Linkage to care following community-based mobile HIV testing compared with clinic-based testing in Umlazi Township, Durban, South Africa. HIV Med. 2014;15:367-372.

9. MacPherson P, Houben RM, Glynn JR, et al. Pre-treatment loss to follow-up in tuberculosis patients in low- and lower-middle-income countries and high-burden countries: a systematic review and metaanalysis. Bull World Health Organ. 2014;92:126-138. 
10. Layer EH, Kennedy CE, Beckham SW, et al. Multi-level factors affecting entry into and engagement in the HIV continuum of care in Iringa, Tanzania. PLoS One. 2014;9:e104961.

11. MacPherson P, Lalloo DG, Webb EL, et al. Effect of optional home initiation of HIV care following HIV self-testing on antiretroviral therapy initiation among adults in Malawi: a randomized clinical trial. JAMA. 2014;312:372-379.

12. Burtle D, Welfare W, Elden S, et al. Introduction and evaluation of a "pre-ART care" service in Swaziland: an operational research study. BMJ Open. 2012;2:e00195.

13. Siedner MJ, Santorino D, Lankowski AJ, et al. A combination SMS and transportation reimbursement intervention to improve HIV care following abnormal CD4 test results in rural Uganda: a prospective observational cohort study. BMC Med. 2015;13:160.

14. McDonald KM, Sundaram V, Bravata DM, et al. Closing the Quality Gap: A Critical Analysis of Quality Improvement Strategies (Vol. 7: Care Coordination). AHRQ Technical Reviews. Rockville, MD; 2007.

15. Craw JA, Gardner LI, Marks G, et al. Brief strengths-based case management promotes entry into HIV medical care: results of the antiretroviral treatment access study-II. J Acquir Immune Defic Syndr. 2008:47:597-606.

16. Gardner LI, Metsch LR, Anderson-Mahoney P, et al. Efficacy of a brief case management intervention to link recently diagnosed HIV-infected persons to care. AIDS. 2005;19:423-431.

17. Bassett IV, Giddy J, Chaisson CE, et al. A randomized trial to optimize HIV/TB care in South Africa: design of the Sizanani trial. BMC Infect Dis. 2013;13:390.

18. Saleebey D. The Strengths Perspective in Social Work Practice. 5th ed. Boston, MA: Allyn \& Bacon; 2009.

19. Katz IT, Bassett IV, Wright AA. PEPFAR in transition-implications for HIV care in South Africa. N Engl J Med. 2013;369:1385-1387.

20. South Africa National Department of Health. Clinical Guidelines for the Management of HIV \& AIDS in Adults and Adolescents. 2010. Available at: http://www.fidssa.co.za/Guidelines/2010_Adult_ART_Guidelines. pdf. Accessed July 2, 2015.

21. South African National Department of Health. The South African Antiretroviral Treatment Guidelines. 2013. Available at: http://www. sahivsoc.org/upload/documents/2013\%20ART\%20Guidelines-Short\% 20Combined\%20FINAL\%20draft\%20guidelines\%2014\%20March\% 202013.pdf. Accessed July 2, 2015.
22. Cornell M, Lessells R, Fox MP, et al. Mortality among adults transferred and lost to follow-up from antiretroviral therapy programmes in South Africa: a multicenter cohort study. J Acquir Immune Defic Syndr. 2014; 67:e67-e75.

23. Willis $\mathrm{S}$, Castel $\mathrm{AD}$, Ahmed $\mathrm{T}$, et al. Linkage, engagement, and viral suppression rates among HIV-infected persons receiving care at medical case management programs in Washington, DC. J Acquir Immune Defic Syndr. 2013;64(suppl 1):S33-S41.

24. Irvine MK, Chamberlin SA, Robbins RS, et al. Improvements in HIV care engagement and viral load suppression following enrollment in a comprehensive HIV care coordination program. Clin Infect Dis. 2015;60:298-310.

25. Gardner LI, Giordano TP, Marks G, et al. Enhanced personal contact with HIV patients improves retention in primary care: a randomized trial in 6 US HIV clinics. Clin Infect Dis. 2014;59:725-734.

26. Finitsis DJ, Pellowski JA, Johnson BT. Text message intervention designs to promote adherence to antiretroviral therapy (ART): a metaanalysis of randomized controlled trials. PLoS One. 2014;9:e88166.

27. Lester RT, Ritvo P, Mills EJ, et al. Effects of a mobile phone short message service on antiretroviral treatment adherence in Kenya (WelTel Kenya1): a randomised trial. Lancet. 2010;376:1838-1845.

28. Pop-Eleches C, Thirumurthy H, Habyarimana JP, et al. Mobile phone technologies improve adherence to antiretroviral treatment in a resourcelimited setting: a randomized controlled trial of text message reminders. AIDS. 2011;25:825-834.

29. Shet A, Costa AD, Kumarasamy N, et al. Effect of mobile telephone reminders on treatment outcome in HIV: evidence from a randomised controlled trial in India. BMJ. 2014;349:g5978.

30. Simoni JM, Huh D, Frick PA, et al. Peer support and pager messaging to promote antiretroviral modifying therapy in Seattle: a randomized controlled trial. J Acquir Immune Defic Syndr. 2009;52:465-473.

31. Dryden-Peterson S, Bennett K, Hughes MD, et al. An Augmented SMS intervention to improve access to Antenatal CD4 testing and ART initiation in HIV-infected pregnant women: a cluster randomized trial. PLoS One. 2015;10:e117181.

32. Bogart LM, Chetty S, Giddy J, et al. Barriers to care among people living with HIV in South Africa: contrasts between patient and healthcare provider perspectives. AIDS Care. 2013;25:843-853.

33. World Health Organization. Global Update on HIV Treatment 2013: Results, Impact and Opportunities. Available at: http://www.who.int/hiv/ pub/progressreports/update2013/en/. Accessed July 2, 2015. 